\title{
Perturbation Theory and Dalgarno-Lewis (DL) Method in Presence of Multiple Laser Beams
}

\author{
N. Shrestha \\ Trichandra Multiple Campus, Tribhuvan University,Kathmandu \\ e-mail:nilamspradhan@gmail.com
}

\begin{abstract}
The evaluation of second and higher order perturbation of energy by iterative solution of Schrodinger's equation, rather than evaluation of matrix element is described.
\end{abstract}

Key words: perturbed hamiltonian, dalgarno operator

\section{Introduction}

While there are many ways to solve Schrodinger equation (SE) analytically, we cannot always obtain an exact solution. Therefore, we must move on to different approaches to obtain more precise results. For example, if the potential energies are known then we can formulate their corresponding Hamiltonians to solve Schrodinger equations very precisely. Hence no such approximations are required for the preceding case. While there are many such Hamiltonians which can be solve fairly easily, there are many more whose solutions can only be approximated numerically. We therefore have to use the widely accepted Perturbation Theory to find approximate solutions of such Hamiltonians_-provided that they are similar to the ones which are fairly easy to solve.

Perturbation theory works best when the system's total energy is “perturbed” by a small additional potential energy (Messsiah 1962). The total Hamiltonian can be expressed as a sum of an unperturbed Hamiltonian

( $\hat{H}_{0}$ ) and a perturbed Hamiltonian $\left(\hat{H}^{\prime}\right)$,

$\hat{H}=\hat{H}+\hat{H}$

The plan is to expand the system of Hamiltonian, and hence the total energy, into a sum of terms involving higher and higher powers of $\hat{H}$, similar to a Taylor series expansion of a function around some value. Since the perturbation is small, we only have to keep the first few terms (typically two or three terms) to obtain a close approximation to the actual total energy.

We must be careful when using the perturbation theory because the perturbing potential should not change the number of bound states of the considered atoms in the system. We can also assume that the unperturbed states of the atoms in consideration form a complete set. We can then express the corrected states as linear combinations of the unperturbed states.

However, if the perturbing potential changes the Hamiltonian of the system such that the number of bound states is increased by one, this new state must have come from the unbounded region. This is a problem because the unbounded region contains a continuum of energies. So while perturbation theory is a very useful tool, it is not always the method of choice.

The time independent (TI) form of the theory is best suited to the problems with discrete energy spectrum. The time dependent (TD) form is useful for problems where there is a continuum of energy states; and here, the product $\mathrm{H} t / \hbar$ must less than unity. In both the forms, the solution is obtained by expanding Eigenvalues and Eigen-functions in a series of small parameters that represents the strength of perturbing terms. The TISE 
Nepal Journal of Science and Technology Vol. 14, No.2 (2013) 165-170

$\hat{H} \Psi=E \Psi$

and for TDSE,

$\hat{H} \Psi(r, t)=i \hbar \frac{\partial \Psi(r, t)}{\partial t}$

The solution of TDSE as,

$$
\Psi(r, t)=\sum C_{n}(t) \psi(r) e^{-t^{r a r}}
$$

Here $C_{0}(t)$ is the expansion coefficient which is our more interest.

From TDSE, and using initial state as ground state

$$
\left(\mathrm{H}_{0} \psi_{n}=\mathrm{E}_{\mathrm{n}} \psi_{n}\right)
$$

Hence, writing in Dirac Notation

$$
i \hbar \sum \frac{\partial C(t)}{\partial t} e^{-t}|\psi(r)\rangle=\sum C(t) e^{\tau^{t} H^{\prime}}|\psi(r)\rangle
$$

Multiplying by bra vector $\left\langle\psi_{m}^{*}\right|$ in both sides and integrating over all the space

$$
\begin{aligned}
& i \hbar C_{m}^{\square}(t)=\sum_{n} C_{n}(t) e^{-\frac{i}{\hbar}\left(E_{n}-E_{m}\right) t} \mathrm{H}_{m n}^{1} \\
& =\sum_{n} C_{n}(t) e^{-i \omega_{n m} t} \mathrm{H}_{m n}^{1} \\
& \text { Where, } \hbar o_{n m}=E_{n}-E_{m}
\end{aligned}
$$

\section{$\hbar \omega_{n m}$ is known as transition energy.}

Assuming the system is in Eigen-state $\left|\psi_{n}\right\rangle$ at $\mathrm{t}=0$, we can express the expansion coefficient for the first order perturbation as

$$
\begin{aligned}
& i \hbar C_{n}(t)=C_{n} e^{-i \omega_{n n^{\prime}}} \mathrm{H}^{1}, m \neq n \\
& C_{a}(t)=(i \hbar)^{-1} \int d t e^{-i a n} \mathrm{H}^{\prime}(t
\end{aligned}
$$

Thus, this equation can be directly integrated, if the perturbed Hamiltonian is known.

Hence the new wave function is known and we can calculate the behavior of the system from this new wave function.

$$
|\Psi\rangle=e^{-\frac{t_{n}{ }^{0}}{t}}\left|\psi_{n}^{0}\right\rangle+\sum C_{n}(t)(t) e^{-\sum_{n} r}\left|\psi_{n}\right\rangle
$$

\section{For the second order}

$$
C_{n}{ }^{(2)}(t)=(i \hbar)^{-1} \sum C_{n}(t) e^{-i w_{n}} \mathrm{H}^{\prime}
$$

For the higher order ( $S^{\prime} t h$ ) of perturbation, the expansion coefficient becomes,

$$
\begin{aligned}
& i \hbar C_{\alpha} \quad(t)=\sum C_{n}(t) e^{-\omega} \mathrm{H}_{w,}^{\prime} \\
& s=0,1,2 \ldots \ldots \text { order of perturbation. }
\end{aligned}
$$

$\mathrm{H}$ ere, $\mathrm{H}_{n=1}^{\prime}$ is the perturbed Hamiltonian in matrix form $\left\langle\psi^{\prime}(r)\left|\mathrm{H}^{\prime}\right| \psi(\gamma)\right\rangle$.

The equation (3) is the set of coupled differential equations for $C_{n}(t)$.

The matrix element $\left\langle\psi^{\prime}(r)\left|\mathrm{H}^{\prime}\right| \psi(r)\right\rangle$ is non zero, and thus we will use the selection rule by which all the matrix elements for given perturbation vanish, except for a few "select" ones characterized by special changes in the quantum numbers.

We now consider a hydrogen atom, and then expose this system to an electric field treated classically in dipole approximation. The electric field alters the Hamiltonian of the initial system, which in turn alters the corresponding Eigen-energies and Eigen-states. Perturbation theory allows us to find approximate solutions to the perturbed Eigen-value problems by beginning with the known exact solutions of the unperturbed problems and then making small corrections to be based on the new perturbing potential.

Then, the perturbation part of the Hamiltonian is

$$
H^{1}=-\sum E_{0} e^{-2\left(\omega+\tilde{\delta}_{j}\right)} \hat{\varepsilon}_{j} \cdot \gamma
$$

Where, $a$ is the angular frequency of the external radiation and $\delta$ the phase difference between different beams and $\widehat{E}_{i}$ represent polarization vector of the different beams are propagating along the same direction. 
Now, we have evaluated the transition between the continuum states of two Eigen states $|g\rangle$ as initial state for $(\mathrm{t}=0)$ and $|f\rangle$ as final states at later time $\mathrm{t}$. Let, ${ }^{\hbar} \omega_{\mathrm{o}}=E_{f}-E_{g}$,

where, $a_{0}$ is the frequency of the transition between two states.

$\hbar$ a $>0$ means atom has absorbsed the photon,

$\hbar a<0$ means atom has emitted the photon.

Plugging equation (4) into equation (1) after performing t integral (Shrestha et al. 2011)

$C^{(1)}(t)$

$$
E_{0}\left\langle f\left|\hat{\sigma}_{j} \cdot r\right| g\right\rangle\left[\frac{e^{(\omega 0+\infty)}-1}{\left(\omega_{0}+\omega\right)}+\frac{e^{(\omega 0-\omega)}-1}{(\omega,-\omega)}\right]
$$

Let the external frequency $\omega$, be nearly equal to the transition frequency $a_{0}$. Then the second term in the above equation becomes arbitrarily large with respect to the first. Still for the fixed time $\mathrm{t}$ and $\mathrm{H}(\mathrm{t})$, since we cannot have $C(t)\rangle 1$. We choose sufficiently small amount of time such that our perturbation expansion works even when is almost equal to. Thus, we can neglect the first term giving

$C$ (f) $\square E_{0}\langle f|\hat{\varepsilon} \cdot r| g\rangle\left[\frac{e^{\left(a_{0}-a\right)}-1}{\left(a_{0}-a\right)}\right]$

The probability of atom at time $t$ is

$$
\mathrm{P}=\left|C^{(1)}(t)\right|^{2}
$$

$\square E_{0}^{2}\left|\left\langle f\left|\hat{\varepsilon}_{i} \cdot r\right| g\right\rangle\right|\left[\frac{e^{\left(\omega_{0} \omega r\right.}-1}{\left(\omega_{0}-a\right)}\right] \mid$

$\mathrm{U}$ sing the identity operator,

$\left|e^{\nu}-1\right|^{2}=2(1-\cos \theta)=4 \sin ^{2} \theta / 2$

Hence, the probability at time $t$ is,

$P(t)$

$\square E_{0}^{2}\left|\left\langle f\left|\hat{\varepsilon}_{i} \cdot r\right| g\right\rangle\right| \frac{\sin ^{2}\left[\left(\omega_{0}-a\right) t / 2\right]}{\left(a_{0}-a\right)^{2}}$

\section{The transition rate}

$\Gamma^{(1)} \square E_{0}^{2}\left|\left(f\left|\hat{\varepsilon}_{i} \cdot r\right| g\right\rangle\right|^{2} \delta\left(\omega_{0}-a\right)$

The first order correction gives the ordinary, linear optical properties of material.

For second or der correction

$C_{f n}^{(2)}(t) \square \int d t \sum_{n} C_{n}^{(1)}(t) e^{-i(t, n)} H^{\perp}$

$\square \int d t E_{0}\left\langle n\left|\hat{\varepsilon}_{k} \cdot r\right| g\right\rangle \times$

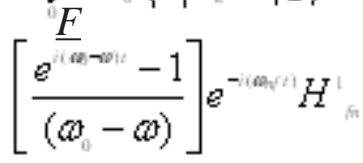

Here, $\hbar \omega_{0}=E_{n}-E_{0}=\hbar \omega_{n}$

$C_{f}^{(2)}(t) \square E_{0}^{2} \frac{1}{(\omega-\omega)}\langle f|\hat{\varepsilon} \cdot r| n\rangle\left\langle n\left|\hat{\varepsilon}_{k} \cdot r\right| g\right\rangle \times$

$\left[\frac{e^{i \omega_{p}-1 \omega}-1}{\left(\omega_{f}-\omega\right)}-\frac{e^{i(\omega)-1)}-1}{\left(\omega_{f}-\omega\right)}\right]$

The probability is

$P^{21}$

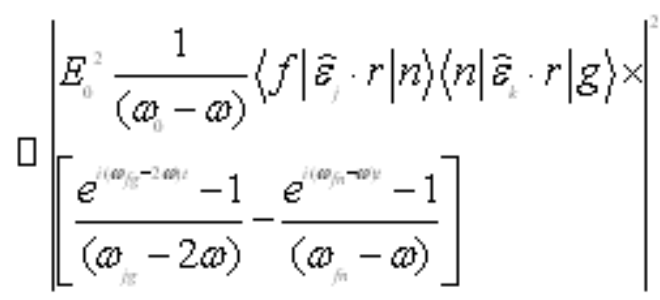

Similarly for three photon, after performing integration,

$C(t)$

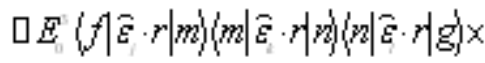

$\left[\frac{e^{\omega}-1}{(\omega-\omega)(\omega-2 \omega)(\omega-3 \omega)}-\frac{e^{\omega}-1}{(\omega-\omega)(\omega-2 \omega)(\omega-\omega)}\right]$ 
The probability of atom in $|f\rangle$ at time t is

$$
\begin{aligned}
& P(t) \\
& \left|\begin{array}{l}
E_{0} \sum \sum \frac{\left\langle f\left|\hat{\varepsilon}_{i} \cdot r\right| m\right\rangle\left\langle m\left|\hat{\varepsilon}_{i} \cdot r\right| n\right\rangle\left\langle n\left|\hat{\varepsilon}_{i} \cdot r\right| g\right\rangle}{(\omega-\omega)(\omega-2 a)} \times \mid \\
{\left[\frac{e^{(a)}-1}{(\omega-3 a)}\right]}
\end{array}\right|
\end{aligned}
$$

The transition $r$ ate for multiple beams $j$, $k$ and $l$, can be expressed as

\section{For first order correction}

$\Gamma, \square E^{2}|\langle f|\hat{\varepsilon} \cdot r| g)|^{2}$

For two and three photons

$\Gamma^{21}$

$\square E{ }_{0,}^{2} E_{0}\left|\sum\left\langle f\left|\hat{\varepsilon}_{i} \cdot r\right| n\right\rangle\left\langle n\left|\hat{\varepsilon}_{i} \cdot r\right| g\right\rangle\left[\frac{1}{\left(\omega_{0}-a\right)}\right]\right|$

$\Gamma^{3} \mathrm{Q}$

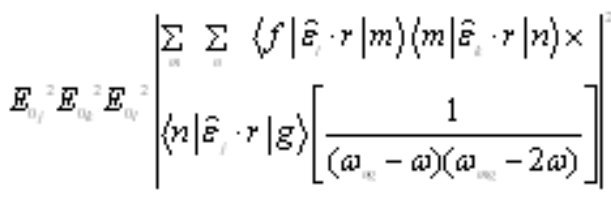

The calculation of ionization $r$ ate has been reducing to a matter of evaluating matrix element.

Letus denote the matrix element of the form by $M$ as

For first order correction

$M^{0},\langle\langle\hat{\varepsilon} \cdot r \mid g\rangle$

For second order correction

$M^{(2)},\left[\frac{\sum\left\langle f\left|\hat{\varepsilon}_{i} \cdot r\right| n\right\rangle\left\langle\left(n\left|\hat{\varepsilon}_{i} \cdot r\right| g\right\rangle\right.}{(a-a)}\right] \ldots \ldots \ldots(0)$

For third or der correction

$M$

$\mathrm{q}\left[\frac{\sum \sum\left\langle f\left|\hat{\varepsilon}_{i} \cdot r\right| m\right)\left(m\left|\hat{\varepsilon}_{i} \cdot r\right| n\right\rangle\left\langle n\left|\hat{\varepsilon}_{i} \cdot r\right| g\right\rangle}{(a-a)(a-2 a)}\right]$.
Dalgarno and Lewis (Dalgamoet al. 1956) developed a simple and practical method to solve perturbation equations; the method was later adopted by many authors to tackle a variety of problems. This method (Francisco 1952) consists of writing the perturbation correction to the Eigen function as

$$
\Psi(r)=F(r) \Psi_{0}(r), p=0,1,2 \ldots \ldots
$$

And solving the resulting equations; the Dalgamo operator $F(r)$ thus becomes,

$-\frac{1}{2} \nabla F-\frac{1}{\Psi} \nabla \Psi \cdot \nabla F+V F-\sum E F=0$

In this equation, $\nabla$ is the gradient vector operator and the dot stand for the standard scalar product. These equations are easier to solve than the original differential equation for the perturbation corrections. In many cases the correction factors $F_{p}$ are simple polynomial function of the coordinates. For $F=1$ is suitable solution to the equation of order zero, and $E$ does not appear in the perturbation equation The Dalgarno-Lewis method (Nandi ef al. 1996) allows us to calculate the perturbation series to higher orders for non degenerate states. The method is based on conversion of Eigen value problem into series of inhomogeneous differential equation These equations determine successively the correction to the Eigen function and the energy correction are obtrined by simple expectation value (Schiff 1968).

In our calculations as shown in equation (5) and equation (6), we have asumed two auxiliary dimensionless operators for two photons as $F_{j}$, and $G$ for three photons, where $j, k$ are the number of beams having same frequency, but different polarization vectors propagating along the same direction

Equation(5) is evaluated by defiring $F$, such as

$\left.(\hat{\varepsilon} \cdot \vec{r})|g\rangle=\left[F H_{v}-H F+a F\right] \mid g\right)$

$(\hat{\varepsilon} \cdot \vec{r}) \psi=[F H-H F+\omega F] \psi$ 
N. Shrestha/Perturbation Theory and Dalgarno......

Now, the equation (5) becomes,

$M_{i}=\left(f\left|\left(\hat{\varepsilon}_{i} \cdot \vec{r}\right) F\right| g\right)$

Here, $H_{0}=-\frac{1}{2} \nabla-\frac{1}{r}$

Thus, $(\hat{\varepsilon} \cdot \vec{r}) \psi$

$=\frac{\nabla F}{2} \psi+\nabla F \cdot \nabla \psi+a F \psi$.

Similarly for three photon ionization, we define $G$ such as

$\left(\varepsilon_{i} \cdot \not \gamma\right) F|g\rangle=\left[G_{p} H_{0}-H_{0} G_{p}+\omega G_{p}\right]|g\rangle$

Then equation (6) becomes,

$M_{i}=\left\langle f\left|(\hat{\varepsilon} \cdot \vec{r}) G_{\mu}\right| g\right\rangle$

$(\hat{\varepsilon} \cdot \vec{r}) F \psi$

$=\frac{\nabla G_{p}}{2} \psi+\nabla G_{\psi} \cdot \nabla \psi+a G_{i} \psi$

In general for the higher order, this can be done by defiring a set of $i-1$ operators $\xi_{i n}$ with $i=$ $1,2, \cdots n-1$ such as (Delone 1999).

$\left(\vec{\varepsilon}_{i} \cdot \vec{\gamma}\right) F_{n+1}|g\rangle=\left(F H_{0}-H_{0} F+n \omega F\right)|g\rangle$

In hydrogen atom, the electron potential is the well known spherically symmetric coulomb potential, owing to the spherically symmetric; it is very convenient to solve TDSE in spherical coordinates (Griffiths 1999).

\section{Let $\quad F=(\vec{\varepsilon} \cdot \vec{r}) f(\omega, \gamma)$}

Where, $(\hat{\varepsilon} \cdot \vec{r})$ is the angular part given by Legendre polynomial and unknown $f(\omega, r)$ simplyas $f$ is the radial part.

After a few manipulations the differential equation for two photon iorization takes the form,

$r f^{\prime \prime}+(4-2 \gamma) f^{\prime}+(2 a \gamma-2) f=2 \gamma$

Similarly for three photon, after assuming

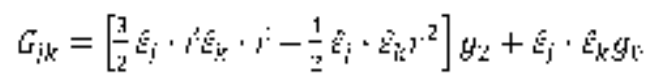

Then after some algebra, we can have the simp. differential equation as

$r g^{\prime \prime}+(2-2 r) g^{\prime}+4 a r=\frac{2}{3} r f$

$r g_{2} "+(6-2 r) g_{2}{ }^{\prime}+(4 a r-4) g=\frac{4}{3} r f$

Here we have used the method of Laplace transfor: (Ince 1956) for the solution of this differential equatic (Radhakrishananet al. 2004).

$\Phi(p, q, \lambda, t, r)=\int d s e^{-r i-1} K(p, q, \lambda, s)$

$K(p, q, \lambda, s)=\left(\frac{1-\lambda}{1+\lambda}\right)^{X}(s+\lambda)^{p /}(s-\lambda)^{-X}$

Finaly, we can have $f(r)$, as

$$
\begin{aligned}
f(r)= & \frac{1}{\omega}-\frac{1}{2 \omega} \Phi(1,1, \lambda, 1, r) \\
g(r)= & \frac{1}{3 \omega^{2}}-\frac{1}{3 \omega^{4}} \Phi(1,1, \lambda, 1, r) \\
& +\frac{2}{3 \omega^{4}} \int d t \frac{K(1, \lambda, 1, t)}{K(3,3, t)} \Phi(2,2, \lambda, t, r)
\end{aligned}
$$

where $\lambda_{1}=\sqrt{(1-2 \omega)}$ and $\lambda_{2}=\sqrt{(1-4 \omega)}$

$g(r)=\frac{2}{3 a^{2}}\left[\frac{r^{2}}{4}+\frac{1}{4 a^{2}}(1-2 \lambda)-\frac{3}{8 a}\right]$

$-\frac{1}{3 \lambda a^{4}} \Phi(0,0, \lambda, t, r)$

$-\frac{1}{6 a^{4}}\left[r^{2}+\frac{\lambda}{a} r-\frac{1}{a}\left(1+\frac{1}{\lambda}\right)\right] \Phi(1,1, \lambda, 1, r)$

$-\frac{1}{3 a}\left(1+\frac{1}{\lambda}\right) \int d \frac{K(1,1, \lambda, 1, t) t}{K(1,1, \lambda, t)}$

$\times\left[\frac{1-2 a}{a(1+\lambda)}-\frac{t}{a}-\frac{2}{t+\lambda}+\frac{2}{(t+\lambda)^{2}}\right] \times$

$\Phi(0,0, \lambda, t, r)$

\section{Results and Discussion}

The idea of DL method is to define an auxiliary operator such that the evaluation of intermediate sum is not 
needed. Now, the difficulties associated with the infinite summation is reduced to the problem of finding an appropriate expressions for the operators $F$ and $G_{i a}$.

We have explored the technique of evaluating higherorder perturbation theory for the multiple beams of same frequency but different polarization from the point of view of Schrodinger's differential equation. The method seems very well adaptable for the problems such as that of the hydrogen atom (Charles 1959).

\section{Acknowledgements}

I would like to thank my supervisors Prof. Dr. J. J. Nakarmi and Prof. Dr. L. N. Jha, for their guidance and encouragement. I express a sincere gratitude to Prof. Ramesh Babu Thauullathil for his fruitful remarks on multiphoton ionization by multiple laser beams, Cochin University of Science and Technology, CUSAT, Kerala, and gratefully acknowledge INSA-NAST bilateral exchange program for funding research at CUSAT, Kerala, India. This work has been supported in part by University Grant Commission, Sanothimi, Bhaktapur.

\section{References}

Charles, S. 1959. Calculation in Schrodinger perturbation theory. Annals of Physics 2: 159-169.
Dalgarno, A. and J.T. Lewis. 1956 . An equivalence of variational and perturbation calculations of small disturbances. Proc. Roy. Soc. A 69: 628-630.

Delone N. B. and V.P. Krainov. 1999. Multiple ionization of atom. In: Multiphoton Processes in Atoms. Springer- Verlag, Berlin, Heidelberg, pp 190-221.

Francisco, M. F. 1952. Perturbation theory in coordinate representation. In: Introduction to Perturbation Theory. CRC Press, Boca Ratan, 13-23.

Griffiths, D. J. 1999. Vector analysis. In: Introduction to Electrodynamics. $3^{\text {rd }}$ edition, Printice Hall of place country-region India Private Lmt, 1-10.

Ince, E. L. 1926. The solution of linear differential equation by definite integral. In: Ordinary Differential Equations. New York, Dover Publications, 187-203pp.

Messsiah, A. 1962. Stationary perturbation theory. In: Quantum Mechanics Volume II.North Holland Publishing Company, Amsterdam, pp 685-739.

Nandi, T.K. and P.K. Bera. 1996. The Dalgarno-Lewis method as small perturbation theory. J. Phys. A. Maths. Gen. 29: 1101-1105.

Radhakrishanan R. and R.B. Thayyullathil 2004. Nonresonant multiphoton ionization in atomic hydrogen. Phy. Rev. A. 69: 033407(1)-033407(5).

Schhiff, L.I. 1968. An alternative treatment of the perturbation series. In: Quantum Mechanics. $3^{\text {rd }}$ edition, Mc Graw Hill Book Company, pp 263-267.

Shrestha, N. and J.J. Nakarmi. 2011. One, two and three photon absorption in atomic hydrogen. Nepal Journal of Science and Technology, 12: 324-329. 